\title{
BMJ Open How do Australian women cope with pelvic girdle pain during pregnancy? A qualitative study protocol
}

\author{
Dragana Ceprnja, ${ }^{1,2}$ Lucinda Chipchase, ${ }^{1,3}$ Pranee Liamputtong, ${ }^{1}$ Amitabh Gupta ${ }^{1,2}$
}

To cite: Ceprnja D, Chipchase L, Liamputtong $\mathrm{P}$, et al. How do Australian women cope with pelvic girdle pain during pregnancy? A qualitative study protocol. BMJ Open 2018;8:e022332. doi:10.1136/ bmjopen-2018-022332

- Prepublication history for this paper is available online. To view these files, please visit the journal online (http://dx.doi. org/10.1136/bmjopen-2018022332).

Received 12 February 2018 Revised 6 June 2018 Accepted 7 June 2018

\section{Check for updates}

C C Author(s) (or their employer(s)) 2018. Re-use permitted under CC BY-NC. No commercial re-use. See rights and permissions. Published by BMJ.

${ }^{1}$ School of Science and Health, Western Sydney University, Penrith, New South Wales, Australia

${ }^{2}$ Physiotherapy Department, Westmead Hospital, Sydney, New South Wales, Australia ${ }^{3}$ University of Canberra, Canberra, Australian Capital Territory, Australia

Correspondence to Dragana Ceprnja; D.Ceprnja@westernsydney. edu.au

\section{ABSTRACT}

Introduction Pelvic girdle pain is commonly experienced during pregnancy and results in significant physical, psychosocial and work-related challenges. Few studies have investigated the lived experiences of pregnant women with pelvic girdle pain and their coping strategies. There is a need to develop a greater understanding of this prevalent condition among Australian women. Thus, this study seeks to gain information about the impact of pelvic girdle pain on daily life and how women cope with this condition during pregnancy.

Methods and analysis A qualitative research design, situated within a phenomenological framework, is adopted. The participants will be invited to describe their lived experiences of pregnancy-related pelvic girdle pain, the impact on their daily life and the strategies they use to cope with the condition. A stratified purposive sample will be undertaken to ensure the sample provides information-rich cases representative of women with pregnancy-related pelvic girdle pain. Face-to-face, individual, semistructured interviews will be conducted with participants at Westmead Hospital, Sydney, Australia. A solicited diary will be offered to any participants who may find attending the interview difficult. All participants will also be invited to attend a focus group session. The different methods of data collection used in this study will allow for triangulation, thereby increasing the trustworthiness of findings.

Ethics and dissemination Ethical approval has been granted by the Human Research Ethics Committees of Westmead Hospital, Sydney, and Western Sydney University, Sydney. Dissemination of results will be via journal articles and conference presentations.

\section{INTRODUCTION}

Pregnancy is a joyful time in most women's lives; however, the physical changes that transform the body create symptoms ranging from mildly annoying to severely disabling. Commonly, pregnant women report morning sickness, heartburn, swelling of the limbs and musculoskeletal pain. ${ }^{1}$ Musculoskeletal pain during pregnancy includes spinal pain with the most common condition being pregnancy-related pelvic girdle pain (PPGP), which is reported to be experienced between the fifth lumbar vertebra and gluteal folds. ${ }^{2}$
Strengths and limitations of this study

This study seeks to provide a greater understanding of the impact of pelvic girdle pain on daily life and how women cope with this condition within the Australian context.

- A diverse and broad sample of women with pregnancy-related pelvic girdle pain (PPGP) will be included, such as those from varying socioeconomic situations and different ethnocultural backgrounds.

- Uses rigorous design and methodology to capture the lived experiences of vulnerable women.

- Will provide knowledge that will inform healthcare professionals and determine the best support(s) needed so that sensitive healthcare practice can be implemented.

- As the study is based on a qualitative approach and given that the participants are from one health facility, the sample may not be considered entirely representative of all women in Australia with PPGP.

Worldwide, the prevalence of PPGP has been reported to range from as low as $7 \%$ to as high as $84 \% .^{2-4}$

Women with PPGP report physical, psychosocial and work-related challenges, with changes to family relationships, altered sleep patterns, impaired mobility and sick leave from work. ${ }^{4-9}$ Additionally, women with PPGP report higher rates of depression and have a lower quality of life than pregnant women without PPGP as measured by the Nottingham Health Profile. ${ }^{4}$ Despite the significant impact on daily life, there has been limited inquiry about how women cope with PPGP during pregnancy and the resources they may use or need to cope.

Coping has been defined as any cognitive or behavioural attempt, successful or unsuccessful, to manage conditions that are perceived as taxing or induce stress. ${ }^{10}{ }^{11} \mathrm{~A}$ recent systematic review of 45 studies synthesised the research describing how adult pregnant women cope with prenatal issues such as stress, depression and smoking. ${ }^{12}$ Of the 45 included studies, only one study investigated 
the theme of coping with lumbopelvic pain in pregnancy. ${ }^{13}$ In a Taiwanese population, Chang et $a l^{13}$ reported that women used a number of coping strategies such as resting, continued performance of undertaking tasks and asking others, such as family, for assistance with their function during third trimester of pregnancy. This finding concurs with other studies included in the systematic review which report that pregnant women use many strategies to cope with various issues in pregnancy, such as stress and depression. ${ }^{12}{ }^{13}$ Chang et al ${ }^{3}$ used a cross-sectional design and employed questionnaires to evaluate how women coped with lumbopelvic pain during pregnancy, however, it did not offer any insight into the lived experiences of women. The quantitative analysis by Chang $e t \mathrm{al}^{13}$ did not allow an exploration describing the strategies used by women to help them cope with PPGP in their daily lives during the third trimester of pregnancy. A qualitative, narrative approach is needed to allow for women's stories to be told in a way that would allow the development of a deeper understanding of their experiences of coping with PPGP.

To date, there has been limited investigation into PPGP using qualitative methodology. A recently published systematic review reported experiences of women with PPGP based on eight studies and described that pain had a major impact on women's lives and families. ${ }^{14}$ However, from the eight studies, only three studies described the experiences of women living with PPGP during pregnancy subsequent to PPGP having been classified by the recommended guidelines. ${ }^{68914}$ These three studies, all conducted in Sweden, reported that women with PPGP struggled with daily life and often avoided movement at home and work. ${ }^{689}$ Further, household tasks, such as childminding, cooking and cleaning, were often redistributed to partners, parents and other relatives. ${ }^{6}$ However, there are limitations associated with these three studies. All of the included participants were living with their partner in these studies, and no details of ethnicity or country of birth of the participants were provided. These are important considerations as both partner status and culture may influence ways of coping and the level of family support and involvement.

Developing a better understanding of how women cope with PPGP from the woman's perspective will help improve the awareness of healthcare professionals who support women during pregnancy. It has been suggested that healthcare professionals view PPGP as a normal consequence of pregnancy and therefore may not be supportive or provide information that is of value to pregnant women who are struggling to cope with PPGP. ${ }^{5891516}$ Information from the woman's perspective will educate healthcare professionals, and raise the awareness of this disabling condition for employers, work colleagues, friends and family members. Developing greater community awareness will more likely allow better support and understanding of women who experience PPGP offering improvement in being able to cope.

This investigation seeks to describe the lived experiences of PPGP among Australian women. An understanding of the impact of PPGP on daily life and how women cope with PPGP will be explored. Knowledge gained from this qualitative enquiry will better inform healthcare practitioners and the women they seek to support during pregnancy.

\section{AIMS AND OBJECTIVES}

The overarching aim of this study is to explore the lived experiences of women living with PPGP. Specifically this study aims to investigate:

1. The impact of PPGP on daily life.

2. Whether women feel they can cope with PPGP.

3. Strategies that women use to deal with PPGP.

4. Support that women may have or need, to help them cope with PPGP.

\section{METHODS AND ANALYSIS \\ Design and setting}

A qualitative study will be performed to provide a rich description of the lived experiences of women living with PPGP. ${ }^{1718}$ This design will enable participants to describe their experiences of how PPGP affects their daily life, the strategies they use to cope with PPGP and the support they may have or require to allow them to cope with PPGP. Qualitative research is essential as we know little about the lived experiences of women with this health condition and this approach offers us the opportunity to obtain detailed descriptions about how women see and think about PPGP. ${ }^{17}$

This study will be situated within a phenomenological framework, as we attempt to understand what the experience means for the person who has lived it; in this case, the lived experience of PPGP. ${ }^{17}$ A phenomenological framework requires the researchers to examine the issue from the individuals who have a first-hand experience. ${ }^{17}$ As the participants in our study are women who are living with PPGP, the phenomenological methodological framework is particularly relevant.

This study will be conducted at Westmead Hospital in Sydney, Australia, from May 2018 to February 2020. Westmead Hospital is a large teaching and tertiary referral government-funded hospital in an urban centre, with over 5700 births recorded annually. ${ }^{19}$ A recent report determined that $23 \%$ of women attending Westmead Hospital suffer from PPGP, and demonstrates that many women are affected. ${ }^{5}$ The hospital has a catchment area which includes women from a diverse range of socioeconomic situations, ethnocultural backgrounds, educational levels and working status.

\section{Participants}

Participants will be recruited from either the antenatal clinic when attending for care during their pregnancy, or from the physiotherapy outpatient clinic when attending for management of pregnancy-related conditions. Potential volunteer participants will be provided 
with information about the aims and methods of the study and be assured that their confidentiality and privacy will be maintained such that no information which may identify them as participants in the study will be disseminated or presented in any form. Potential volunteer recruits will also be informed that they are free to withdraw from the study at any time without their antenatal care or physiotherapy care being affected. Those agreeable to participating will provide written and informed consent.

To be included in this study, women will have to be over 18 years of age, between 14 and 38 weeks' gestation, classified with PPGP and have a sufficient command of the English language in order to be able to provide written and informed consent and complete the interview. Attempts shall be made to include culturally and linguistically diverse women who speak English in order to ensure we hear a range of voices, including those from ethnic backgrounds who otherwise may lack the opportunity to have a voice. ${ }^{17}$ It has been reported that women from ethnic backgrounds are less willing and able to participate in research. ${ }^{1}$ To attempt to overcome this, this study design uses a flexible and culturally sensitive approach to include women who may be vulnerable or difficult to include due to a lack of transport.

All participants will be classified as having PPGP according to recommended guidelines, ${ }^{20}$ which include the report of pain distal to fifth lumbar vertebra and between the posterior iliac crest and the gluteal fold with or without pubic symphysis pain; report of pain during two or more of the following activities including walking, standing, climbing a flight of stairs, turning over in bed or getting out of a chair; at least two physical examination tests (posterior pelvic pain provocation test, ${ }^{21}$ palpation of long dorsal sacroiliac ligament, ${ }^{22}$ active straight leg raise test $\mathrm{t}^{23}$ and modified Trendelenburg test ${ }^{24}$ ) being positive on the side of the reported symptoms.

Exclusion criteria will include the self-report of any medical or obstetric complication(s) which affects pregnancy, such as serious pathology of non-musculoskeletal origin including pre-eclampsia, eclampsia, serious intellectual or psychiatric impairment, systemic disease(s), or recent spinal fracture, trauma or surgery.

A stratified purposive sample will ensure that the sample will be representative of women with PPGP who attend Westmead Hospital. ${ }^{25}{ }^{26}$ For example, a diverse and broad sample of women from different self-reported ethnocultural backgrounds, those who have a partner and those who do not, and women participating in varying levels of exercise and physical activity will be sought. Potential participants who are less than 33 weeks' gestation will be given up to 1 month to consider their involvement in this research project. Those who are beyond 33 weeks' gestation will be given an appropriate time frame under 1 month to consider their involvement in order to meet the inclusion criteria of being up to 38 weeks' gestation.

\section{Procedure}

The researcher (the first author) will contact each participant to schedule a single, face-to-face interview at a mutually convenient date and time at Westmead Hospital. All participants will receive reimbursement of transport or parking costs to the value of $\$$ A20.00 to attend the interview. Participants will be asked to complete a written questionnaire to determine anthropomorphic characteristics (age, height, body mass) and information about their current pregnancy, such as the weeks of gestation, parity, pregnancy type (singleton, multiples) and current pain level using a visual analogue scale. ${ }^{27}$ Information about their sociodemographic background, country of birth, self-identified ethnicity, marital status, current physical activity level and work status will also be collected. This questionnaire will be completed in entirety just before the interview. The primary investigator will analyse this information to provide a profile of the sample population and this information may guide further, purposive sampling with the aim to ensure that the total sample recruited for this study is representative of the broader characteristics of the women who attend the hospital for care for PPGP.

An interview guide consisting of open-ended questions will be used with a flexible and responsive approach. The interview guide will ensure that the same range of topics will be discussed with each participant with the questions aiming to determine the lived experience of having PPGP. Women will be asked about how PPGP impacts their daily life, such as describing what changes to the performance of daily activities they have made, and how they feel they cope, including what strategies they use to help them deal with PPGP. Women's thoughts about what other support(s) or information should be provided to help them to better cope with PPGP will also be determined.

The interview will be digitally recorded (details of the instrument). The interviewer will also make written notes during and after each interview which may include observations, thoughts and ideas about the interview and other information about the participants' responses. ${ }^{28}$

Participants who are unable to attend an interview will be offered an alternative method of providing information with a solicited diary so that they can record their day-to-day experiences living with PPGP. Participants who consent to complete a diary will be provided a set of written questions in order to keep the purpose of the research in mind. Women will be instructed to write as much as they can about whatever comes to mind in response to the interview guide over a period of 1 week. Each participant can write diary entries at any time of day, which is an important consideration in this group which may include women who have a disturbance to their normal daily routine. Pregnancy can be a busy time for many women, with work and/or family commitments limiting their available spare time. Therefore, the use of a diary offers a flexible approach that may better suit some women and, in particular, allows for the recruitment of vulnerable groups who may not otherwise volunteer to participate. ${ }^{29}$ On completion of the diary, these women 
will be offered another opportunity for a face-to-face interview in case they would like to discuss any items in detail and in person with the researcher. Similarly, all women who attended the face-to-face interview will be offered to complete a diary to be able to provide more information over a period of 1 week. Therefore, it is possible some of the participants may complete both an interview and a diary in either order.

The interviews will be transcribed verbatim. All participants will be provided with the opportunity to review and edit their interview and/or diary responses. According to the 'member checking method', ${ }^{17}$ the participants should be invited to review their interview transcripts to see if we have recorded what they said correctly or not. If the participants feel that the transcript contains information that they do not feel comfortable for use, they have the right to edit the interviews. The researchers will endeavour to keep the time between completion of the interview or diary and receipt of the transcript for review to 2 weeks.

Following the face-to-face interviews and submission of completed diaries, all participants will be invited to a focus group session in order to explore whether any new information is raised by participants. The focus groups will consist of six to eight women with PPGP and will be conducted by the researcher who conducted all the face-to-face interviews and instructed participants on the completion of a diary. Offering participants the option to attend a focus group will enable and allow for interaction between participants to explore and clarify their personal responses, attitudes and points of view and gives the participants different methods to tell their story. ${ }^{17}$ Responses in the group setting may differ from those provided in individual interview or diary methods. Alternatively, responses may be reinforced in the group setting. Together, the different methods (individual and group) allow for development of concordance between the responses with different data collection methods. ${ }^{17}$

\section{Patient and public involvement}

There was no patient or public involvement in the design of this protocol.

\section{Sample size}

The sample size required will be determined when saturation of themes is achieved. Saturation is defined as when the collection of new data does not shed any further light on the issue under investigation. ${ }^{17} 30$ Research situated within the phenomenology methodology has suggested that this requires between 5 and 25 participants. ${ }^{31}$ In this study, we will aim to interview at least 25 women to ensure richness of the data with a broad and diverse sample and to provide confidence that saturation will be reached. However, we cannot be sure that no new themes would arise if data collection was continued after this point.

\section{Data analysis}

Thematic analysis will be performed where each recorded interview will be listened to several times to make sense of the data and the interview as a whole. ${ }^{17}{ }^{32}$ Similarly, diary entries will be read and read again a number of times to make sense of the entries as a whole. Initially, open coding will be conducted by naming chunks of the participants' narratives in the text. ${ }^{33}$ Frequent discussions between all authors will ensure thorough and consistent coding patterns emerge from the data. Codes will then be grouped to form meaningful categories. To enhance data credibility, the analysis will be critically discussed and compared among all authors, hence moving back and forth between the entire text and categories. Further, analysis of three transcripts will be conducted by two authors independently to see if similar categories emerge. The next step will be to construct broader themes from the categories (the NVivo V.10 software will be used to assist at this stage). An audit trail will also be adopted to ensure the rigour of the study. The authors will provide detailed clarification of the reasons for theoretical, methodological and analytic choices that will allow readers to see how such decisions were made and the reasons for making them. ${ }^{17}$

\section{Data management}

Data including consent forms, questionnaires, interview notes and diaries will be stored in a locked drawer of a filing cabinet within the Physiotherapy Department at Westmead Hospital. Electronic data, including information from questionnaires, digital recordings and interview transcriptions, will be stored on a password-protected desktop computer within the Physiotherapy Department at Westmead Hospital. Only the research team will have access to the data. Data will be stored in a reidentifiable manner where all participants will be assigned a coded number and pseudonym. No participant names or other identifying features will appear in any form of data reporting. Instead, pseudonyms will be used to identify quotes or comments made by interviewees. The code will be kept in a locked drawer of a filing cabinet within the Physiotherapy Department at Westmead Hospital and will only be able to be accessed by a member of the research team. The information will be stored for a period of 5 years as consistent with NSW Health guidelines.

\section{ETHICS APPROVAL AND DISSEMINATION}

Ethical approval has been granted by the Human Research Ethics Committees of Westmead Hospital, Sydney (AU RED LNR/17/WMEAD/508), and Western Sydney University, Sydney (RH12532). All study participants will provide written informed consent and will receive written information about the study, including the contact details of the Westmead Hospital Human Research Ethics Committee, so that they are able to report any concerns or complaints about the study. 
The study team will disseminate project outcomes via publication, conference presentations, workshops and webinars.

\section{DISCUSSION}

This project will provide information about the lived experiences of women living with PPGP and the challenges they face in order to determine how women cope with PPGP. The project will fill a gap in the literature and add conceptual understanding about the lived experience of PPGP among Australian women of which little is known. It is contended that a greater understanding of the impact of PPGP on daily life, together with information about the strategies women use to help cope with PPGP, will provide information for healthcare providers to offer sensitive and effective support to pregnant women in this phase of their life. In addition, knowledge gained will increase the broader community awareness of the burden PPGP may cause to society.

The findings of this project may lead to follow-up research to investigate novel ways of providing support to women with PPGP, and improving pathways of care, including but not limited to education and referral to other services, to help women effectively manage this condition. Further, research aimed at quantifying the cost to society that PPGP poses may also be considered to determine the size of the burden associated with this significant health issue.

Contributors DC, LC, PL and AG were involved in the design of the trial. DC drafted the manuscript. LC, PL and AG edited the manuscript. All authors have approved the final version.

Funding The authors have not declared a specific grant for this research from any funding agency in the public, commercial or not-for-profit sectors.

Competing interests None declared.

Patient consent Not required.

Ethics approval Westmead Hospital, Sydney, Western Sydney Local Health District Human Research Ethics Committee.

Provenance and peer review Not commissioned; externally peer reviewed.

Open access This is an open access article distributed in accordance with the Creative Commons Attribution Non Commercial (CC BY-NC 4.0) license, which permits others to distribute, remix, adapt, build upon this work non-commercially, and license their derivative works on different terms, provided the original work is properly cited, appropriate credit is given, any changes made indicated, and the use is non-commercial. See: http://creativecommons.org/licenses/by-nc/4.0/.

\section{REFERENCES}

1. National Collaborating Centre for Women's and Children's Health (UK). Antenatal care: routine care for the healthy pregnant woman. London: RCOG Press, 2008.

2. Kanakaris NK, Roberts CS, Giannoudis PV. Pregnancy-related pelvic girdle pain: an update. BMC Med 2011;9:15.

3. Bastiaanssen JM, de Bie RA, Bastiaenen $\mathrm{CH}$, et al. Etiology and prognosis of pregnancy-related pelvic girdle pain; design of a longitudinal study. BMC Public Health 2005;5:1.

4. Van De Pol G, Van Brummen HJ, Bruinse HW, et al. Pregnancyrelated pelvic girdle pain in the Netherlands. Acta Obstet Gynecol Scand 2007;86:416-22.
5. Pierce H, Homer CS, Dahlen HG, et al. Pregnancy-related lumbopelvic pain: listening to Australian women. Nurs Res Pract 2012;2012:1-10.

6. Persson M, Winkvist A, Dahlgren L, et al. "Struggling with daily life and enduring pain": a qualitative study of the experiences of pregnant women living with pelvic girdle pain. BMC Pregnancy Childbirth 2013;13:111.

7. Olsson C, Nilsson-Wikmar L. Health-related quality of life and physical ability among pregnant women with and without back pain in late pregnancy. Acta Obstet Gynecol Scand 2004;83:351-7.

8. Elden H, Lundgren I, Robertson E. Life's pregnant pause of pain: pregnant women's experiences of pelvic girdle pain related to daily life: a Swedish interview study. Sex Reprod Healthc 2013;4:29-34.

9. Elden H, Lundgren I, Robertson E. The pelvic ring of pain: Pregnant women's experiences of severe pelvic girdle pain: An interview study. Clin Nurs Stud 2014;2:30-41.

10. Lazarus RS, Folkman S. Stress, appraisal, and coping. New York: Springer, 1984

11. Lazarus RS. Coping theory and research: past, present, and future. Psychosom Med 1993;55:234-47.

12. Guardino CM, Schetter CD, Dunkel Schetter C. Coping during pregnancy: a systematic review and recommendations. Health Psychol Rev 2014;8:70-94.

13. Chang HY, Yang YL, Jensen MP, et al. The experience of and coping with lumbopelvic pain among pregnant women in Taiwan. Pain Med 2011;12:846-53.

14. Mackenzie J, Murray E, Lusher J. Women's experiences of pregnancy related pelvic girdle pain: A systematic review. Midwifery 2018;56:102-11.

15. Vermani E, Mittal R, Weeks A. Pelvic girdle pain and low back pain in pregnancy: a review. Pain Pract 2010;10:60-71.

16. Ceprnja D, Chipchase L, Gupta A. The role of physiotherapy in managing pregnancy related pelvic girdle pain. $N Z J$ Physiother 2017;45:56-7.

17. Liamputtong P. Qualitative research methods. 4th edn. Melbourne: Oxford University Press, 2013.

18. Neergaard MA, Olesen F, Andersen RS, et al. Qualitative description - the poor cousin of health research? BMC Med Res Methodol 2009;9:52.

19. The year in review. http://www.wslhd.health.nsw.gov.au/ ArticleDocuments/1232/WSLHD_year-in-review_2016-17.pdf.aspx (accessed 13 Nov 2017)

20. Vleeming A, Albert HB, Ostgaard HC, et al. European guidelines for the diagnosis and treatment of pelvic girdle pain. Eur Spine $J$ 2008;17:794-819.

21. Ostgaard HC, Zetherström G, Roos-Hansson E. The posterior pelvic pain provocation test in pregnant women. Eur Spine $J$ 1994;3:258-60.

22. Albert $H$, Godskesen M, Westergaard J. Evaluation of clinical tests used in classification procedures in pregnancy-related pelvic joint pain. Eur Spine J 2000;9:161-6.

23. Mens JM, Vleeming A, Snijders CJ, et al. Reliability and validity of the active straight leg raise test in posterior pelvic pain since pregnancy. Spine 2001;26:1167-71.

24. Albert HB, Godskesen M, Westergaard JG. Incidence of four syndromes of pregnancy-related pelvic joint pain. Spine 2002;27:2831-4.

25. Palinkas LA, Horwitz SM, Green CA, et al. Purposeful Sampling for Qualitative Data Collection and Analysis in Mixed Method Implementation Research. Adm Policy Ment Health 2015;42:533-44.

26. Patton M. Qualitative research and evaluation methods. 5th edn. Thousand Oaks CA: Sage, 2015.

27. Wewers ME, Lowe NK. A critical review of visual analogue scales in the measurement of clinical phenomena. Res Nurs Health 1990;13:227-36.

28. Cormack DFS. The research process in nursing. 4th edn. Oxford: Blackwell Science, 2000.

29. Liamputtong P. Researching the vulnerable: a guide to sensitive research methods. London: Sage, 2007.

30. Charmaz K. Constructing grounded theory: a practical guide through qualitative analysis. London: Sage, 2006.

31. Creswell J, design R. Qualitative, quantitative and mixed methods. 4th edn. Thousand Oaks CA: Sage, 2014.

32. Braun V, Clarke V. Using thematic analysis in psychology. Qual Res Psychol 2006;3:77-101.

33. Corbin J, Strauss A. Basics of qualitative research: techniques and procedures for developing grounded theory. 4th edn. Thousand Oaks CA: Sage, 2014. 